\title{
Hemoptysis as a first symptom of endoleak after thoracic endovascular aortic repair, which caused aortic rupture and required complex management
}

\author{
Tomasz Synowiec ${ }^{1}$, Marcin Warot ${ }^{1}$, Paweł Burchard², tukasz Paschke' ${ }^{1}$, Zuzanna Łysiak ${ }^{1}$, Paweł Chęciński ${ }^{1}$ \\ 1Department of General and Vascular Surgery and Angiology, Poznan University of Medical Sciences, Poznan, Poland \\ 2Division of Cardiology - Intensive Therapy, Department of Internal Medicine, Poznan University of Medical Sciences, Poznan, Poland \\ Videosurgery Miniinv 2013; 8 (2): 178-181 \\ DOI: $10.5114 /$ wiitm.2011.33453
}

\begin{abstract}
Thoracic endovascular aortic repair (TEVAR) has become the most common procedure in the treatment of thoracic aorta aneurysms. However, potential long-term complications of this technique could be life-threatening. Hemoptysis is a common symptom of lung tumor, especially cancer. On the other hand, hemoptysis can also be caused by bronchitis, tuberculosis, mycosis, and trauma. In this case report, we present a patient with hemoptysis and lung tumor suggesting lung cancer, which was a unique symptom of type IA endoleak after TEVAR and led to rupture of the thoracic aneurysm. It was decided to perform next an endovascular procedure due to the severe state of the patient. Next the thoracotomy was performed because drainage of the left pleural cavity was unsuccessful. In the last stage bronchoscopy was needed to remove the thrombus, which occluded the left main bronchi. Successful management has led to the patient's full recovery. Despite justified popularity of endovascular procedures in the treatment of thoracic aorta aneurysms, we should remember about potential long-term complications. Hemoptysis could be a unique symptom of the endoleak after TEVAR and treatment of such complications could be complex and demanding.
\end{abstract}

Key words: aneurysm, stent graft, thoracic aorta, endovascular repair.

\section{Introduction}

Thoracic endovascular aortic repair (TEVAR) has become the most common procedure in the treatment of thoracic aorta aneurysms. Despite justified popularity, we should remember about potential long-term complications of this technique, which could be life-threatening [1]. Hemoptysis is a common symptom of lung tumor, especially cancer. On the other hand, hemoptysis can also be caused by bronchitis, tuberculosis, mycosis, and trauma [2].

In this case report, we present a patient with hemoptysis and lung tumor suggesting lung cancer, which was a unique symptom of type IA endoleak after TEVAR and led to thoracic aneurysm rupture.

\section{Case report}

A 63-year-old man was admitted to the Department of General and Vascular Surgery due to severe hemorrhagic shock. Concomitant diseases included uncontrolled arterial hypertension and spondylosis. In 2006 the patient underwent TEVAR because of the symptomatic aneurysm of the descending aorta with pleural effusion. In 2009, the second TEVAR was performed due to rupture of the descending aorta below 
the previous stent graft. The last control contrastenhanced computed tomography (angio-CT), which was performed in the outpatient clinic in September 2011, did not reveal any graft-related complications. The aneurysmal sac even shrank in comparison to the previous angio-CT.

In January 2012, the patient suffered from severe hemoptysis. A chest X-ray and a lung CT were performed. They revealed a lung tumor infiltrating one of the bronchi and bleeding to the pulmonary alveoli, with a suggestion of lung cancer (Photo 1). Just before a planned biopsy the patient collapsed. On the basis of the next angio-CT and and chest X-ray, the lung tumor was diagnosed as the cause of the bleeding from the ruptured thoracic aorta aneurysm due to an endoleak type $\mathrm{IA}$, with concomitant massive hemothorax (Photo 2). The patient was intubated and admitted to the Intensive Care Unit. It was decided to perform the next TEVAR due to the severe state of the patient. Two hours after admission, endovascular treatment was performed. This third graft covered the left subclavian artery to ensure a proper proximal landing zone and ended in a distal part of the thoracic aorta. During the operation, drainage of the left pleural cavity was performed. After 5 days of intensive care, there was no satisfactory recovery. Patient was still intubated, and oxygen saturation was only $80 \%$, despite ventilation with $70 \%$ oxygen. The drainage of the left pleural cavity was unsuccessful. Tracheotomy was performed, and a tracheal tube inserted. Repeated X-rays and angio-CT revealed compression atelectasis of the left lung, and massive thrombus in the left pleural cavity.

It was decided to perform a thoracotomy. During the operation, a massive mass $(2000 \mathrm{ml})$ from the left pleural cavity was removed. It consisted of thrombus from fresh bleeding and old thrombus from the aneurysmal sac. The thrombus $(300 \mathrm{ml})$ from the left lung was also removed. The ventilation parameters improved. The patient was conscious but still needed respiratory support. After 4 days, bronchoscopy was performed and a thrombus which occluded the left main bronchi was removed. After this procedure, fast recovery was observed. In 2 days, the patient was breathing by himself without any support, and the tracheal tube was removed. Covering the left subclavian artery caused no symptoms. A control chest $X$-ray, performed 10 days after the procedure, revealed no hemothorax and a satisfactory level of left lung ventilation (Photo 3). After
2 weeks of rehabilitation, the patient was discharged from the hospital in a favorable condition. A followup, performed 6 months after the procedure with angio- $\mathrm{CT}$, revealed no endoleaks and a satisfactory level of left lung ventilation (Photo 4). No signs of graft infection were visible, probably due to long antibiotic therapy.

\section{Discussion}

Since 1953, the thoracic aorta aneurysm could be successfully treated with resection and synthetic

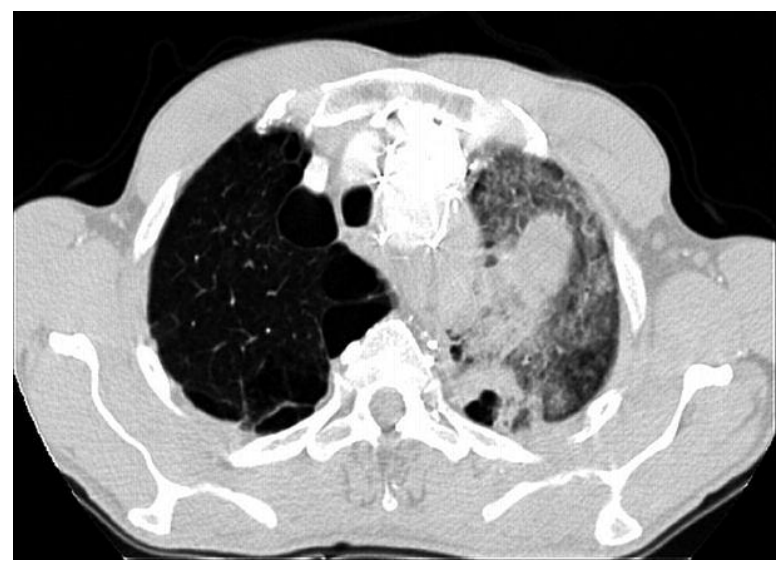

Photo 1. Lung tumor infiltrating one of the bronchi and bleeding to the pulmonary alveoli

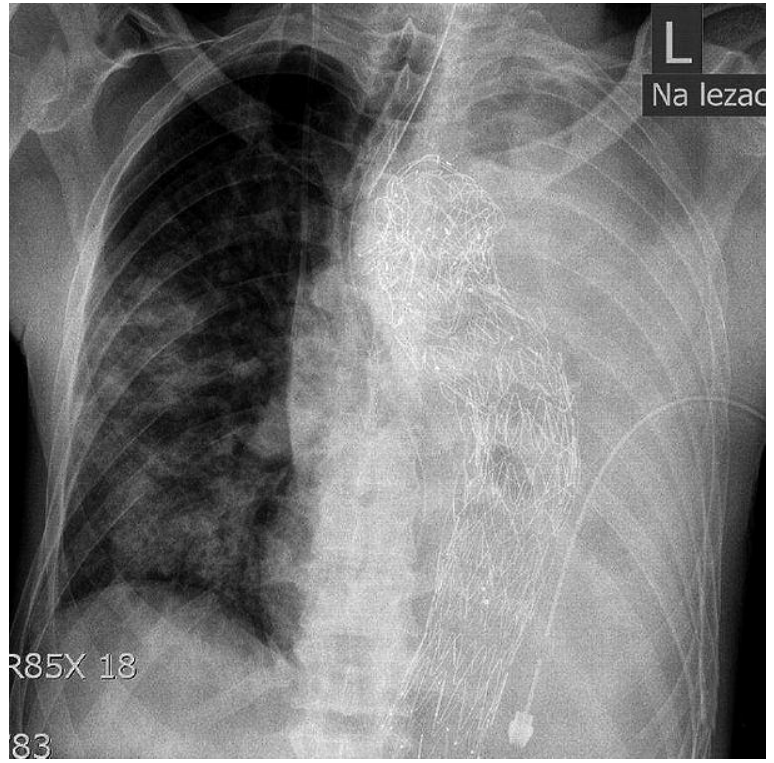

Photo 2. Massive hemothorax, as the result of the bleeding from the ruptured aneurysm due to an endoleak type IA, after thoracic endovascular aneurysm repair 


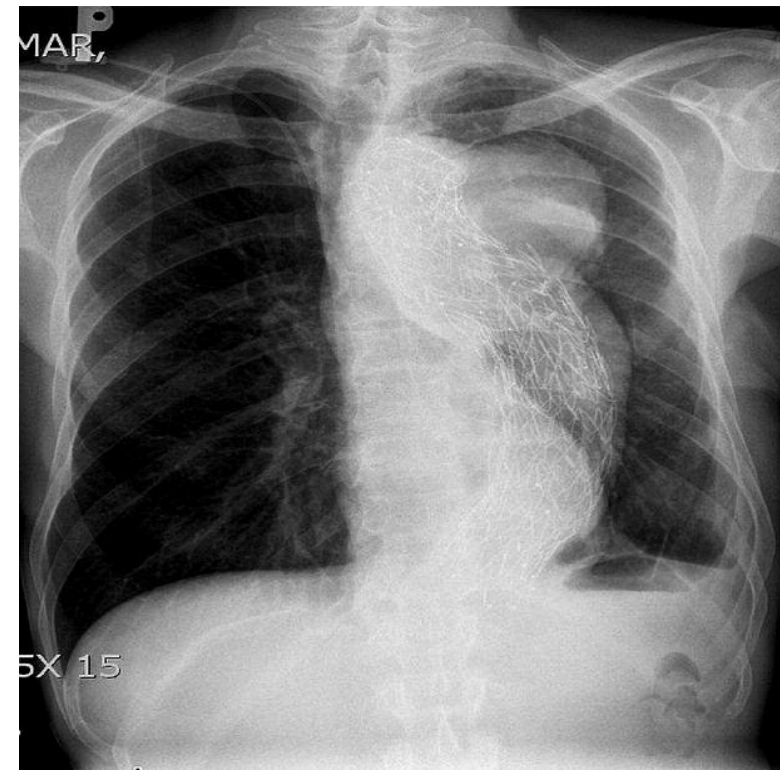

Photo 3. Control chest $X$-ray, performed 10 days after the procedure, revealed no hemothorax and a satisfactory level of left lung ventilation

graft replacement [3]. The other method of treatment is TEVAR, first described by Dake et al. in 1994 [4]. Thoracic endovascular aortic repair has become the most common procedure in the treatment of thoracic aorta aneurysms $[1,5]$.

The most common complications after TEVAR are endoleaks. As far as we know, endoleaks are mainly asymptomatic, and patients after TEVAR require accurate follow-up. In rare cases, despite repeated angio-CT, they may remain undetected, until they rupture [6]. Because of that, any symptom that may suggest such disease should not be overlooked. In the present case, endoleak type IA caused expansion of the aneurysmal sac and probably small rupture to the left lung, which manifested as hemoptysis. Next, because of enlarging endoleak and uncontrolled hypertension, the patient suffered rupture of the aneurysmal sac to the left pleural cavity. In most cases, rupture of the thoracic aorta aneurysm is lethal $[7,8]$. In this patient, probably due to previous adhesions in the pleural cavity, the rupture resulted only in hemorrhagic shock.

Type IA endoleak is rare and means blood flow into the aneurysm sac due to incomplete seal or ineffective seal at the proximal part of the graft. This type of endoleak usually occurs in the early course of treatment [9]. In the present case, this complication occurred atypically a few years after the last stent-

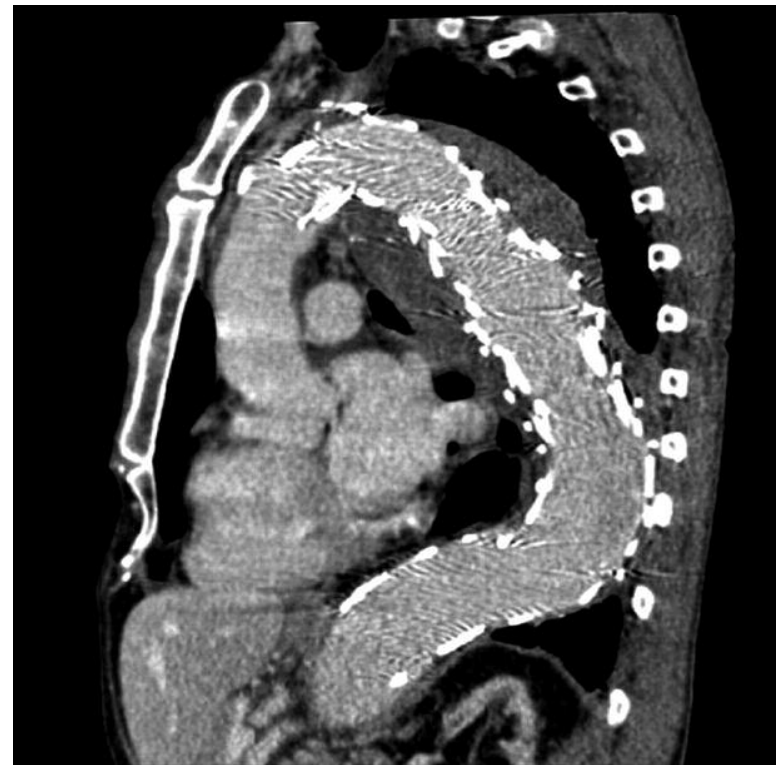

Photo 4. Follow-up performed 6 months after the procedure with angio-CT did not reveal any stent-graft related complications

graft implantation and suddenly, despite regular follow-up.

The third stent-graft placement required a proximal landing zone. The only possibility was to cover the origin of the left subclavian artery. According to the literature, it is performed in up to $40.8 \%$ of cases. Revascularization of the left subclavian artery is not mandatory [10]. Only symptomatic patients, who suffer hand or brain ischemia, require surgical revascularization [11]. In the present case, blood pressure on the left arm was sufficient. Assessment of potential brain ischemia in the intubated patient was difficult, but performed transcranial Doppler examination revealed sufficient blood flow in the cerebral arteries.

The main problem after successful TEVAR was poor ventilation due to unsuccessful drainage of the left pleural cavity. We tried to avoid surgery, but the need for thoracotomy became obvious. During the left lateral thoracotomy, the thrombus from the pleural cavity was removed. It was atypical because, in most cases, blood in the pleural cavity does not create a thrombus. Probably it was an old thrombus from the aneurysmal sac or the mutilated lung initiated clot formation, which led to insufficient drainage. In the case of bronchoscopy, there was a risk of pneumothorax due to the destroyed part of the left lung and previous thoracotomy [12]. Fortunately, there was no 
such complication and ventilation improved, followed by extubation.

In conclusion, hemoptysis could be a unique symptom of endoleak after TEVAR. Treatment of this complication can be complex and demanding.

\section{References}

1. Weidenhagen R, Bombien R, Meimarakis G, et al. Management of thoracic aortic lesions - the future is endovascular. Vasa 2012 41: 163-76.

2. Jougon J, Ballester M, Delcambre F, et al. Massive hemoptysis: what place for medical and surgical treatment. Eur J Cardiothorac Surg 2002; 22: 345-51.

3. DeBakey ME, Cooley DA. Successful resection of aneurysm of thoracic aorta and replacement by graft. J Am Med Assoc 1953; 152: 673-6.

4. Dake MD, Miller DC, Semba CP, et al. Transluminal placement of endovascular stent-grafts for the treatment of descending thoracic aortic aneurysms. N Engl J Med 1994; 331: 1729-34.

5. Mitchell ME, Rushton FW Jr, Boland AB, et al. Emergency procedures on the descending thoracic aorta in the endovascular era. J Vasc Surg 2011; 54: 1298-302.

6. Fattori R, Russo V, Lovato L, et al. Endovascular management of thoracic aortic aneurysms. Cardiovasc Intervent Radiol 2011; 34: 1137-42.

7. Johansson G, Markström U, Swedenborg J. Ruptured thoracic aortic aneurysms: a study of incidence and mortality rates. J Vasc Surg 1995; 21: 985-8.

8. Synowiec T, Chęciński P, Samolewski P, et al. Hybrid procedure in a patient with symptomatic thoraco-abdominal aneurysm and prior abdominal aortic reconstruction - case report. Videosurgery Miniinv 2012; 7: 132-6.

9. Morales JP, Greenberg RK, Lu Q, et al. Endoleaks following endovascular repair of thoracic aortic aneurysm: etiology and outcomes. J Endovasc Ther 2008; 15: 631-8.

10. Kotelis D, Geisbüsch P, Hinz U, et al. Short and midterm results after left subclavian artery coverage during endovascular repair of the thoracic aorta. J Vasc Surg 2009; 50: 1285-92.

11. Desai ND, Pochettino A, Szeto WY, et al. Thoracic endovascular aortic repair: evolution of therapy, patterns of use, and results in a 10-year experience. J Thorac Cardiovasc Surg 2011; 142: 587-94.

12. Navsaria PH, Vogel RJ, Nicol AJ. Thoracoscopic evacuation of retained posttraumatic hemothorax. Ann Thorac Surg 2004; 78: 282-5.

Received: 24.10.2012, revised: 3.02.2013, accepted: 3.02.2013. 